\title{
Experimental Solution to the Laplace Equation, a Tutorial Approach
}

\author{
G. Moradi ${ }^{1}$, M. Marvasti ${ }^{2}$ \\ Department of Electrical Engineering, Amirkabir University of Technology, Tehran, Iran ${ }^{1,2}$
}

\begin{abstract}
In this paper, experimental solutions of the electromagnetics Laplace equation are presented. First, the papers review the Laplace problem, then solve it using analytical, numerical and experimental methods and compare them together. It also shows that how useful information can be obtained from a raw Laplace equation data. Several tutorial measurements and discussions have been proposed. This paper can be used as a good educational tool in Electromagnetic and physics courses.
\end{abstract}

Keywords: Laplace equation, boundary value problems, Electrostatic, PDE, experimental setup.

\section{INTRODUCTION}

In recent years, the development of electromagnetics has shown the impressive importance of the static solution of the electromagnetics problems. On the other hand static solution in the electromagnetics is the basis of the dynamic solution to the electrodynamics problems. So without concentrating on the electrostatics, electrodynamics understanding seems to be improbable. Laplace and Poisson equations are electrostatics problems and the simplest form of the elliptic partial differential equations (PDEs) [1]. In fact the general theory of the Laplace equation solution is known as the potential theory. The solution to this equation is known as harmonic functions [2], and these functions are notable in different fields such as: Electrostatic, Astronomy, Fluid Dynamics, etc.

Numerical solution of linear PDEs can be performed by different methods including finite difference method (FDM), finite element method (FEM), and method of Moments (MoM) [3]. The practical solutions of some types of applied PDEs have been reported in early and recent papers $[4,5]$.

Here, in this paper, a very simple set up for experimental solution of Laplace equation is introduced. The practical results are compared with those of numerical and analytical methods. The most important feature is the tutorial achievements of the results. Some of the most important results are argued. These results can be included in the applied electromagnetics courses to strengthen the understanding of the electrostatic potential concept.

\section{DERIVATION OF LAPLACE EQUATION}

As we know the curl of the static electrical field is zero so it is known as the gradient of a scalar potential V:

$$
\nabla \mathrm{V}=-\mathrm{E}
$$

On the other hand from the differential form of the Gauss rule, the following results can be obtained:

$$
\begin{gathered}
\nabla . \mathrm{E}=\rho / \varepsilon \rightarrow \nabla \cdot \nabla \mathrm{V}=-\rho / \varepsilon \\
\nabla^{2} \mathrm{~V}=-\rho / \varepsilon
\end{gathered}
$$

Equation (3) is known as the Poisson equation. In this equation, $\nabla^{2}$ is the Laplacian operator, $\mathrm{V}$ is the scalar potential, $\rho$ is the charge density and $\varepsilon$ is the permittivity coefficient of the medium. If the amount of $\rho$ in a portion of the medium equals to zero, which is the common and normal case, then this equation is known as the Laplace equation.

Keeping this in mind, in the next section of this paper, numerical solution to the Laplace equation is reviewed. Then the experimental solution to a 2D Laplace problem is studied. Some useful results are discussed; and a comparison between analytical, numerical and experimental solutions for this problem is performed in section IV. The paper ends with a conclusion in section V.

\section{NUMERICAL SOLUTION TO THE LAPLACE EQUATION}

Due to the development of numerical methods in various fields, these methods have become more widespread and various software tools analyze the PDE problems based on these methods. Among the most important of these methods FEM, FIT, MOM, FDM can be mentioned [3]. Also in the fields of electrostatics and electrodynamics, some popular commercial software products exist that use numerical solution methods. For example, COMSOL uses FEM, CST employs FIT, FEKO is based on MOM, HFSS works with FEM.

In this paper, only FDM is used, which is very simple compared with the MOM and the other methods. This method is based on an approximation of the differential operators. One of these approximations i.e. the forward difference (FD) scheme for the first order differential equation is as follows. 
Vol. 5, Issue 9, September 2016

$$
\frac{\partial}{\partial x} V(x, y, z) \approx \frac{V(x+\Delta x, y, z)-V(x, y, z)}{\Delta x}
$$

$$
V(x, y, z)=\frac{1}{4 \pi R^{2}} \oiint V(x, y, z) d s
$$

Similarly, the backward difference (BD) approximation for the second order differentiation is as follows:

$$
\frac{\partial^{2} \mathrm{~V}}{\partial \mathrm{x}^{2}} \approx \frac{\frac{\partial}{\partial \mathrm{x}} \mathrm{V}(\mathrm{x}, \mathrm{y}, \mathrm{z})-\frac{\partial}{\partial \mathrm{x}} \mathrm{V}(\mathrm{x}-\Delta \mathrm{x}, \mathrm{y}, \mathrm{z})}{\Delta \mathrm{x}}
$$

Combining (4) and (5), the following result can be obtained for the $2^{\text {nd }}$ order derivative.

$$
\begin{aligned}
& \frac{\partial^{2}}{\partial x^{2}} V(x, y, z) \\
& \approx \frac{V(x+\Delta x, y, z)-2 V(x, y, z)+V(x-\Delta x, y, z)}{\Delta x^{2}}
\end{aligned}
$$

For the $\mathrm{z}$ and $\mathrm{y}$ dimensions, similar equations can be obtained. Employing these equations we are able to approximate a PDE and reduce the order of its complexity from a differential equation to an algebraic equation. Equation (3) can be written as follows.

$$
\begin{array}{r}
\nabla^{2} \mathrm{~V}=\frac{\partial^{2}}{\partial \mathrm{x}^{2}} \mathrm{~V}(\mathrm{x}, \mathrm{y}, \mathrm{z})+\frac{\partial^{2}}{\partial \mathrm{y}^{2}} \mathrm{~V}(\mathrm{x}, \mathrm{y}, \mathrm{z}) \\
+\frac{\partial^{2}}{\partial \mathrm{z}^{2}} \mathrm{~V}(\mathrm{x}, \mathrm{y}, \mathrm{z})=0
\end{array}
$$

So after replacing (6) and the similar equations for other dimensions into (7) the following equation is obtained.

$$
\begin{aligned}
v(x, y, z)=\frac{1}{6}[v( & x+\Delta x, y, z)+v(x-\Delta x, y, z) \\
& +v(x, y+\Delta y, z) \\
& +v(x, y-\Delta y, z) \\
& +v(x, y, z+\Delta z) \\
& +v(x, y, z-\Delta z)]
\end{aligned}
$$

This equation says that the voltage at a point $(x, y, z)$ can be considered as the average of voltages of the surrounding points. This fact is known as the "mean value" theorem. Also for a $2 \mathrm{D}$ problem where $\frac{\partial^{2}}{\partial z^{2}} V(x, y, z)=0$, (8) can be written as the following equation.

$$
\begin{aligned}
v(x, y)=\frac{1}{4}[v(x & +\Delta x, y)+v(x-\Delta x, y) \\
& +v(x, y+\Delta y) \\
& +v(x, y-\Delta y)]
\end{aligned}
$$

Equations (8) and (9) are the numerical approximation of an exact mean value theorem as follows [3]:

$$
V(x, y)=\frac{1}{2 \pi r} \oint V(x, y) d l
$$

Similarly the continuous form of the 3D mean value theorem is:
By considering the mentioned explanations a MATLAB program can be written for calculation of potentials in all points of an arbitrary structure with an arbitrary boundary conditions. To do this, the following steps must be followed. First we need to discrete the structure. There are different grids for various structures that can be used, such as rectangular grid, skewed grid, circular grid, triangular grid and so on [3]. In this paper, for the sake of simplicity, we consider the rectangular. Then using the methods mentioned in this section, an algebraic equation governing the problem is obtained from its PDE.

Finally by writing these equations for each point of the grid of the structure and putting them into a system of equations, the potential of the unknown points can be calculated. It should be noted that the methods of solving the obtained algebraic system of equations, has a lot of diversity and details. However we simply use the "inv" command in MATLAB software for our program.

\section{EXPERIMENTAL SOLUTION FOR THE 2D LAPLACE EQUATION}

To perform an experimental analysis for a 2D Laplace problem, first the desired structure or container must be physically implemented as shown in Fig. 1. Then the container is filled with a liquid -such as distilled water- to be able to measure voltage of each point of the grid. Then after connecting signal generator to the boundaries, the voltage of each point of the grid of the structure is measured with an oscilloscope or voltmeter. The data are written down and finally the obtained results must be analyzed.

In our 2D Laplace problem, we consider a rectangular structure that we connect $100 \mathrm{mv}$ voltage signals into two of its boundaries (boundaries 1 and 2 in Fig. 2) and zero Volt voltage signal to the other boundaries.

Table 1 shows the voltage of the points of the grid of the structure which is measured experimentally. Fig. 3 shows the MATLAB software analysis over our measured data.

This problem is also solved with analytical and numerical methods. Fig. 4 shows the numerical results obtained by FDM method and Fig. 5 shows analytical results which is calculated by the following equation.

$$
\begin{aligned}
V(x, y)=\sum_{\alpha=1}^{\infty}\left(\frac{200}{\alpha \pi}\left(1-(-1)^{\alpha}\right) \sin (\alpha \pi / 20 y)\right. \\
\quad \times\left(-\operatorname{coth}\left(\frac{3 \alpha \pi}{2}\right)\right. \\
\quad \times \sinh (\alpha \pi / 20(30-x)) \\
+\cosh (\alpha \pi / 20(30-x)))
\end{aligned}
$$


International Journal of Advanced Research in Computer and Communication Engineering ISO 3297:2007 Certified

Vol. 5, Issue 9, September 2016

TABLE I EXPERIMENTAL DATA FOR THE VOLTAGES OF 140 POINTS OF THE GRID OF THE STRUCTURE (472 POINTS MEASURED AT 1 KHZ FREQUENCY).

\begin{tabular}{|c|c|c|c|c|c|c|c|c|c|c|c|c|c|}
\hline 75 & 85 & 90 & 90 & 90 & 90 & 90 & 90 & 90 & 90 & 90 & 90 & 95 & 95 \\
\hline 32 & 52 & 62 & 70 & 76 & 78 & 80 & 85 & 85 & 85 & 85 & 90 & 95 & 95 \\
\hline 12 & 28 & 44 & 54 & 60 & 60 & 60 & 64 & 68 & 68 & 74 & 76 & 80 & 95 \\
\hline 10 & 22 & 38 & 44 & 52 & 58 & 60 & 64 & 64 & 68 & 70 & 78 & 80 & 90 \\
\hline 6 & 16 & 28 & 34 & 40 & 46 & 50 & 56 & 58 & 60 & 64 & 74 & 78 & 90 \\
\hline 6 & 12 & 23 & 30 & 36 & 40 & 44 & 48 & 52 & 56 & 60 & 68 & 76 & 85 \\
\hline 4 & 10 & 18 & 23 & 28 & 33 & 37 & 39 & 42 & 46 & 50 & 58 & 70 & 85 \\
\hline 3.5 & 7.5 & 11 & 16 & 20 & 23 & 27 & 29 & 32 & 35 & 39 & 50 & 60 & 80 \\
\hline 2 & 4.5 & 7.5 & 10 & 12 & 14 & 16 & 18 & 20 & 23 & 27 & 30 & 42 & 76 \\
\hline 1.2 & 1.2 & 5 & 7.5 & 7.5 & 8.5 & 9.5 & 10 & 11 & 12 & 16 & 18 & 25 & 68 \\
\hline
\end{tabular}

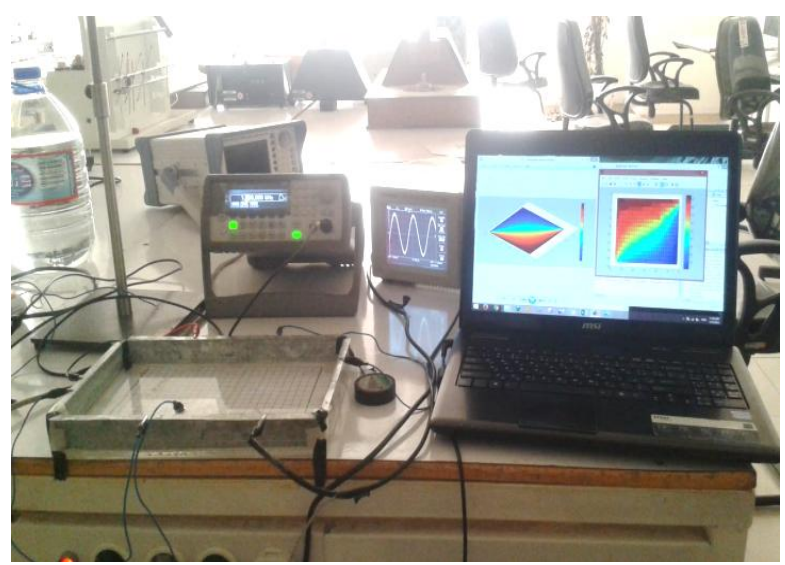

Fig. 1. Experimental set up, implemented for 2D Laplace problem.

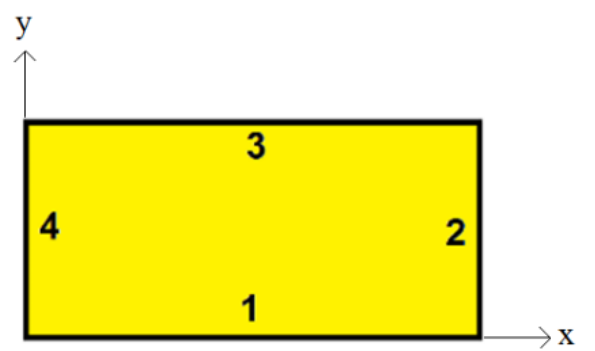

Fig. 2. Boundaries allocation of the structure

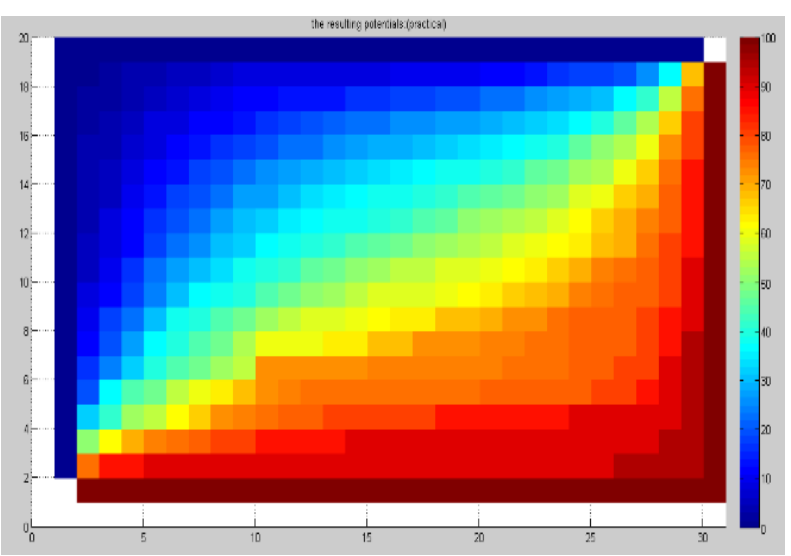

(a)

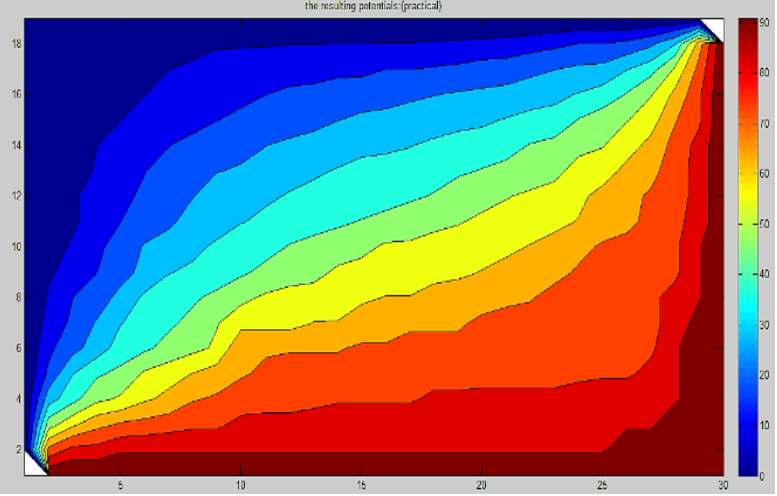

(b)

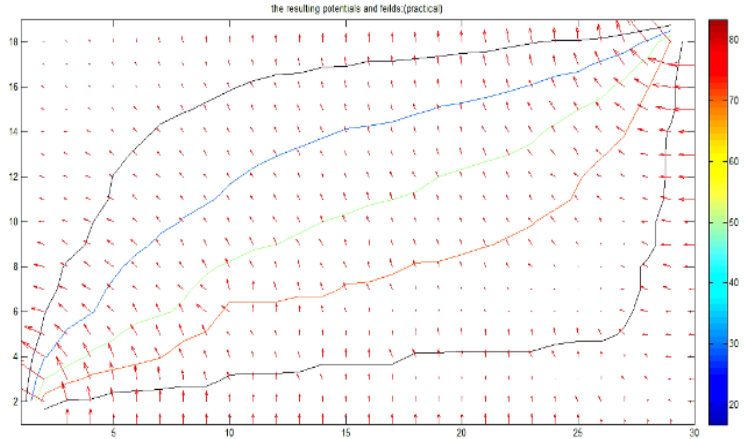

(c)

Fig 3.MATLAB software results over the measured data. (a) The exact voltages measured at each point. (b) Approximate voltages (c) The electric field lines.

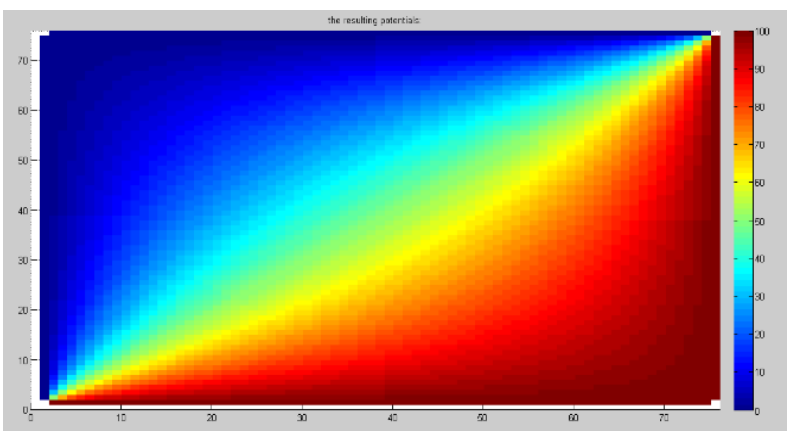

(a) 
Vol. 5, Issue 9, September 2016

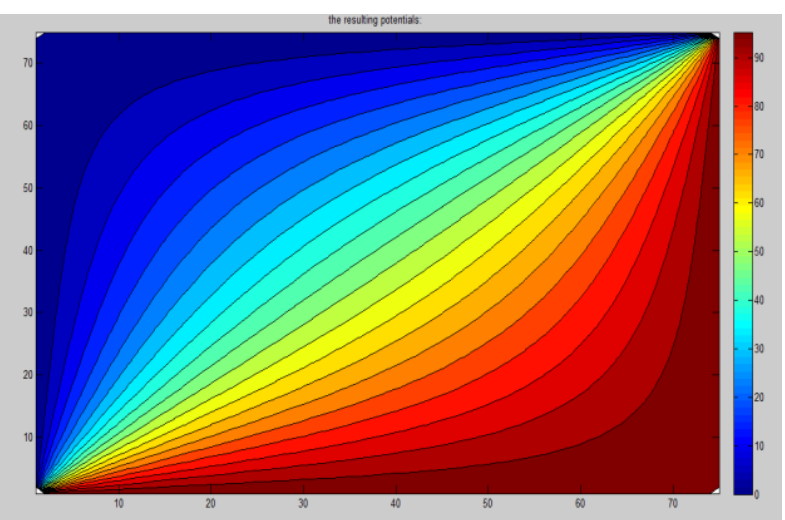

(b)

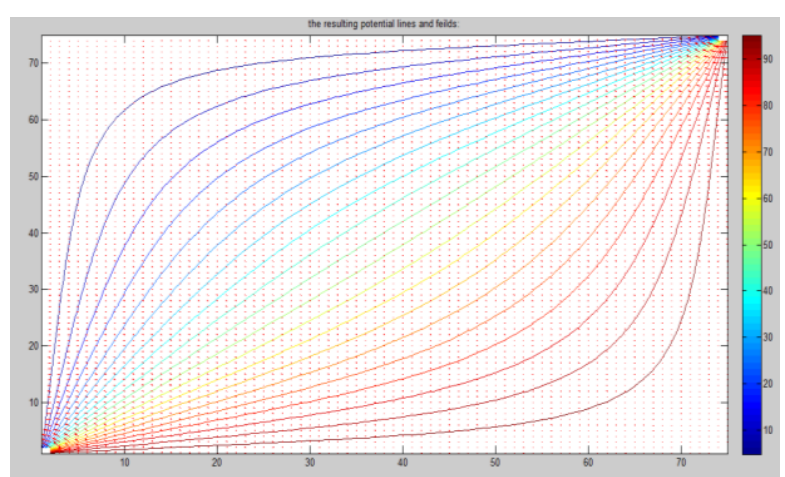

(c)

Fig. 4.Numerical solution of Laplace equation using finite difference routine. a) Continuous format b) continuous format together with equipotential lines c) equipotential lines.

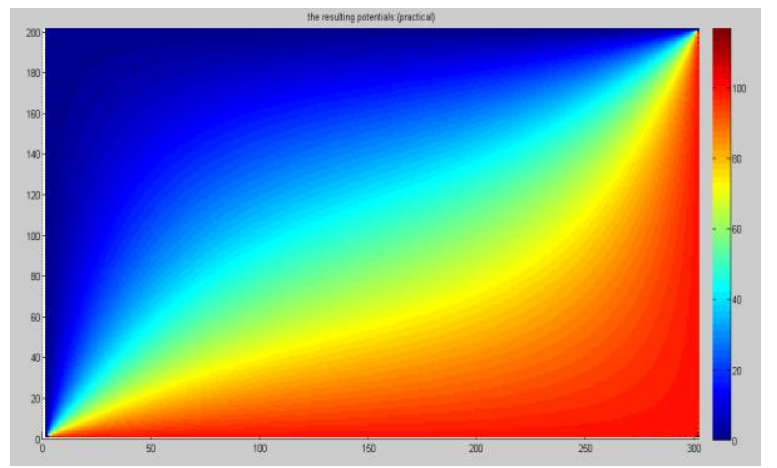

(a)

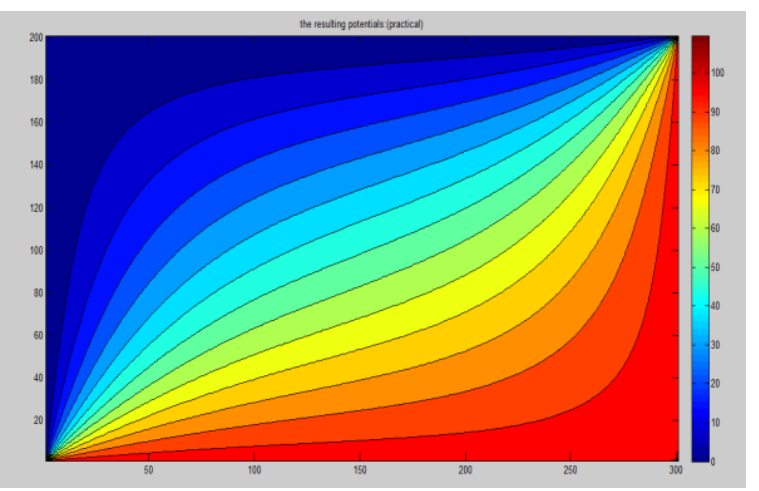

(b)

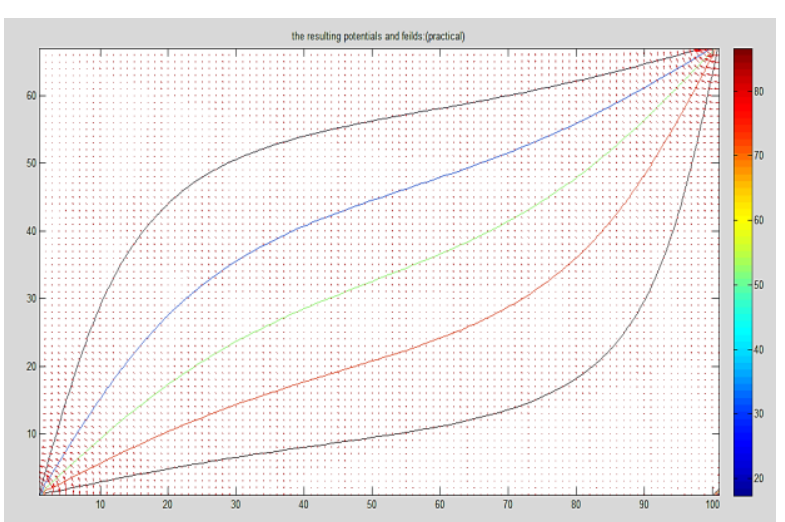

(c)

Fig. 5. Analytical solution of Laplace equation. a) continuous format $b$ ) continuous format together with equipotential lines c) equipotential lines.

The capacitance of this structure can be calculated using the Laplace data obtained. To do that the following equations must be noticed.

$$
\begin{gathered}
C=\frac{q}{v} \\
q=\oint D . d s \\
D=\varepsilon_{\text {liquid }} E \rightarrow D=80 * \varepsilon_{0} * E \\
\oint D . d s \approx 80 * h \times \varepsilon_{0} \\
\times\left(\sum_{\text {on widt } h} E_{x}\right. \\
\left.+\sum_{\text {on lengt } h}\right)
\end{gathered}
$$

MATLABin which $h$ is height of the container or the capacitor. From MATLAB software calculations for experimental method, the following values are obtained:

$$
\begin{aligned}
& \sum_{\text {on widt } h} E_{x}=184 \text { and } \sum_{\text {on lengt } h} E_{y}=220 \\
& C=80 * 0.02 m \times 8.85 * 10^{-12} \times 404= \\
& 0.72 \times 80 p F=57.6 p F
\end{aligned}
$$

It should be noted that the values of electrical fields have been obtained from numerical derivative of voltage function.

Similar to the mentioned method, for numerical and analytical solutions, capacitance value for the structure can be obtained. Capacitance using numerical solution is 63.814 PF and that based on the analytical data is $75.31 P F$. The results are so promising. In the above mentioned calculations, based on the data sheet, the relative dielectric constant of water is assumed to be 80 .

It is also important to check the effect of some of the experimental parameters such as frequency, liquid depth and liquid type on our results.

To check the influence of excitation frequency, we have to note that due to the phenomenon of electrolysis in water or other polar liquids, unlike the numerical and analytical 
Vol. 5, Issue 9, September 2016

solutions, the experimental solution to Laplace equation cannot be done in DC frequency. However if nonpolar liquids are used in the experimental solution, the frequency can be set to zero. Considering temporal form of voltage as $V=A \cos (\omega t)$, from the wave equation in 1 $\mathrm{KHz}$ frequency we have the following results.

$$
\begin{gathered}
\nabla^{2} V=\frac{-\omega^{2}}{c^{2}} A \cdot \cos (\omega t) \rightarrow \frac{10^{6}}{9 * 10^{16}} \cong 0 \rightarrow \\
\nabla^{2} V=0
\end{gathered}
$$

So for low frequency conditions the wave equation approximately is as the Laplace equation. From this equation it can be understood that the lower the frequency that is set, the more accurate results are must be obtained, but based on our measurements that has been done for 100 $\mathrm{Hz}$ and $500 \mathrm{~Hz}$ frequency, the mean squared error (MSE) for $500 \mathrm{HZ}$ is lower than that of the $100 \mathrm{~Hz}$. As mentioned, reason for that is the phenomenon of electrolysis in water.

To check the influence of liquid depth in the conditioner, we have to note that unlike numerical and analytical solutions, in experimental solution an exact 2D problem cannot be solved and only 3D problems can be solved. To reduce the $3 \mathrm{D}$ problem to a $2 \mathrm{D}$ problem, i.e. to remove the voltage variations along the third dimension from the structure, the structure size must be unbounded in this dimension. So by increasing the depth of the liquid, the measured results are more accurate and the effect of the probe depth can be ignored. But building such a big structures or doing measurement in them is quite impossible, so for decreasing the value of electrical field in third dimension instead of making unbounded structure the high permittivity liquids can be used as following equations show.

$$
\begin{gathered}
D_{n 1}-D_{n 2}=\rho \\
\rho=0 \rightarrow D_{n 1}=D_{n 2} \rightarrow \varepsilon_{1} E_{n 1}=\varepsilon_{2} E_{n 2}
\end{gathered}
$$

Therefore;

$$
\text { if } \varepsilon_{1} \gg \varepsilon_{2} \text { then } E_{n 1} \ll E_{n 2}
$$

The relation (20) is written at the boundary of free space and the liquid, so in this equation medium 1 is the liquid and medium 2 is the free space.

Results and Discussions

From the results obtained with experimental, numerical and analytical solution to this Laplace problem we can conclude the following tutorial results.

1) Near the boundaries, the equipotential lines that voltage is constant along them, follow the shape of the boundary.

2) The potential values in the structure are between the maximum and minimum potential values of the boundaries and do not have local maximums and minimums. This validates the mean value theorem.

3) The electrical field lines are perpendicular to the boundaries near them.
4) The electrical field lines start from positive voltages and end with negative voltages.

5) The electrical field lines are stronger near the connection between boundaries having different voltages. This effect is usually known as the edge effect.

6) Mean value theorem certification for an arbitrary point in experimental method. For example for a point whose adjacent voltages are measured as 85, 42, 50 and $70 \mathrm{mV}$, the measured voltage is $62 \mathrm{mV}$. The following calculation shows how accurate the mean value theorem can be practically verified:

$V_{\text {center }}=\frac{1}{4} \times(85 m v+42 m v+50 m v+70 m v)=$ $61.75 m v \approx 62 m v$

7) The stronger the electrical field lines, the more compact the voltage lines Become.

8) It should be noted that our boundaries in our tests are the equipotential boundaries while the problem may have a float (Neumann) boundary. In that case, the formation of the voltage lines and electrical field lines are reversed in comparison with equipotential (Dirichlete) boundary near the boundary. As Fig. 6 shows, the equipotential lines are parallel to the Dirichlete boundaries(boundaries 1 and 3), whilst they are perpendicular to Neumann boundaries (boundaries 2 and 4). This matter is just the opposite for electric field lines.

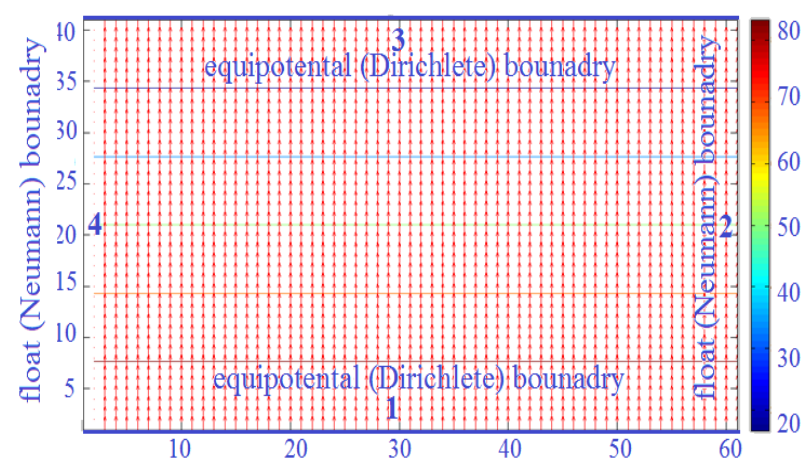

Fig. 6 Comparison between float and equipotential boundaries. The equipotential lines are parallel to the Dirichlete boundaries, whilst they are perpendicular to Neumann boundaries. The electric field lines are shaped just the opposite.

9) From Fig. 6, it can be easily understood that floating the boundaries in two opposite sides is equivalent to removing the dependency of the volatge on that dimension.

10) Using numerical approach, solving the Poisson equation with arbitrary boundary conditions and arbitrary charge density distribution is quite simple. Fig. 7 shows an example of numerical solution to an arbitrary Poisson equation. Interested researchers may develop a test set-up for this case.

11) As mentioned, due to the phenomenon of electrolysis in polar liquids such as water, the experimental solution to Laplace equation cannot be done in zero frequency and DC condition.

12) The higher the permittivity of the liquid the more accurate the Laplace equation will be solved. 
Vol. 5, Issue 9, September 2016

13) The more the liquid depth, the more accurate the Laplace equation solution will be.

14) Also it is possible to check the effect of applying an arbitrary voltage function as a boundary condition. This idea is a little bit complicated to be implemented as an experimental solution, but with the numerical or analytical solution it is easy to achieve the correct answer. Fig. 8 shows the numerical solution to Laplace equation for an arbitrary voltage function as a boundary condition.

15) Also it is possible to check the effect of applying boundary condition with odd or even symmetry. For our scenario (Fig. 2), in odd symmetry, boundaries 1 and 3 must have voltages with opposite signs. Similarly for even symmetry, those boundaries must have the same voltages. Boundaries 2 and 4 in both cases are floated. The numerical solution for these conditions is shown in Fig. 9.

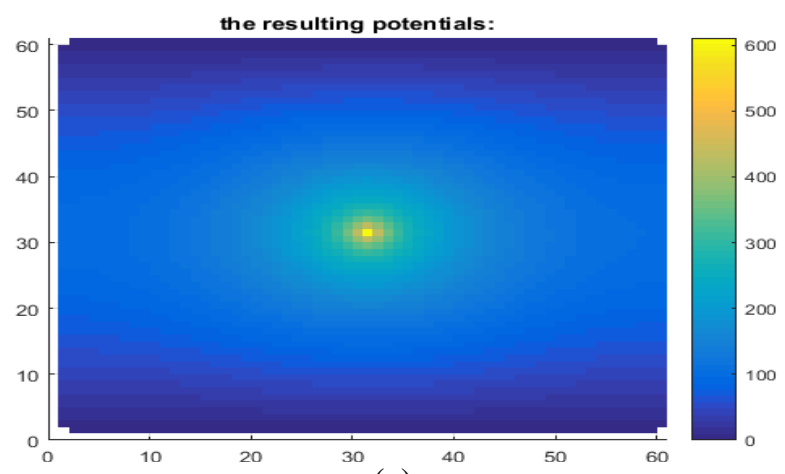

(a)

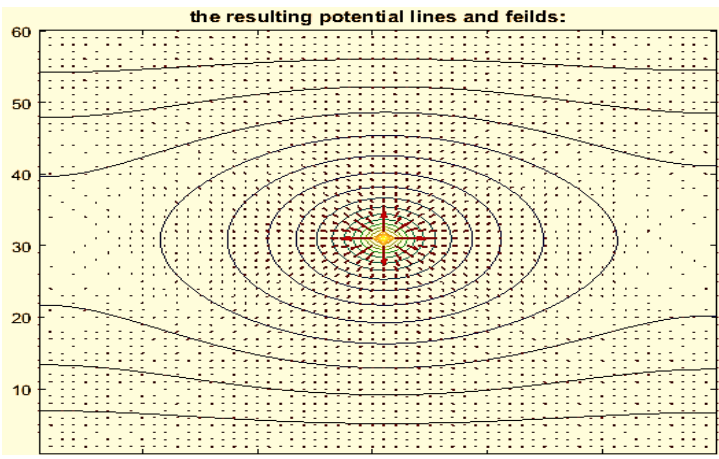

(b)

Fig. 7. Numerical solution to an arbitrary Poisson equation. (a) Exact value of voltages (mV). (b) Electrical field lines and equipotential lines.

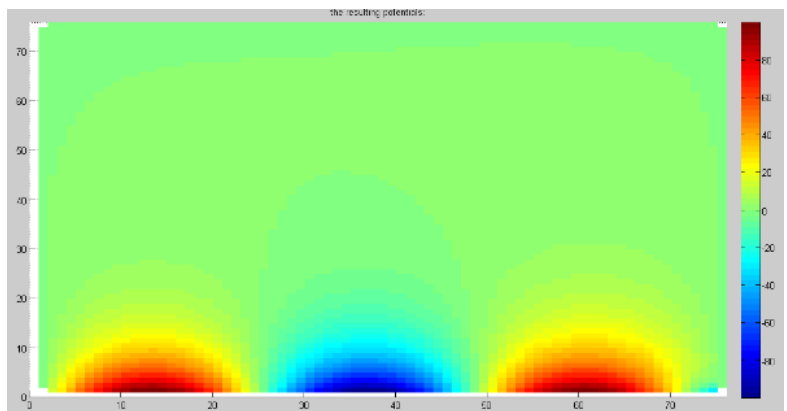

Fig. 8. Numerical solution to Laplace equation for an arbitrary voltage functions as first boundary condition.

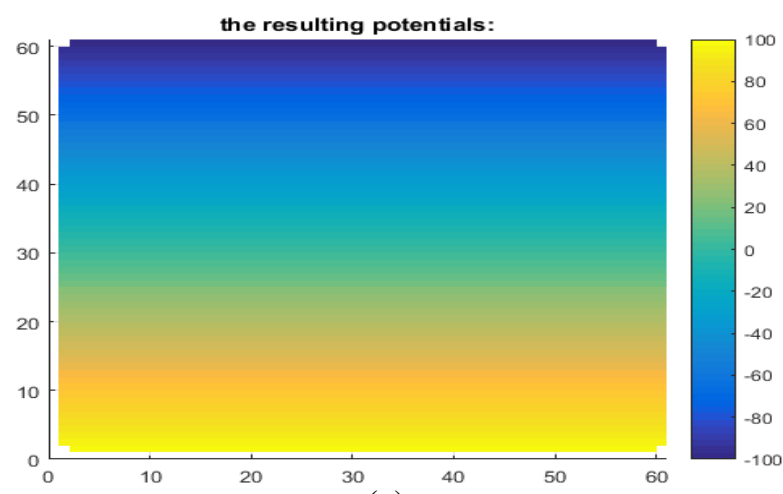

(a)

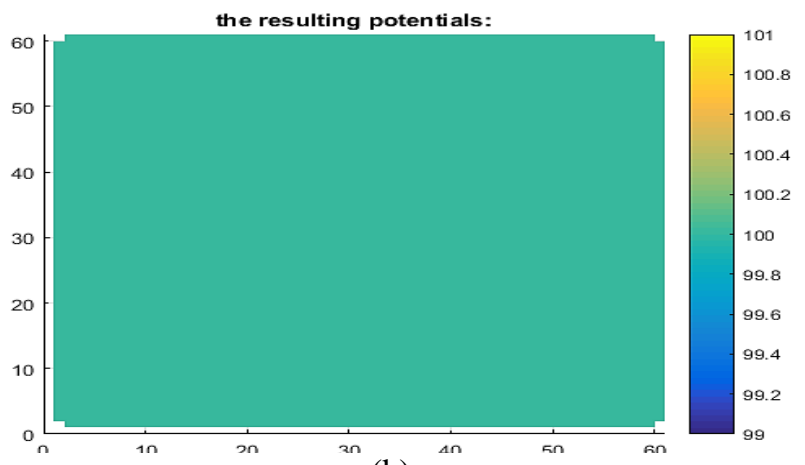

(b)

Fig. 9.Numerical solution for odd and even symmetry of voltage of the boundaries. (a) Odd symmetry in which obviously voltages in the middle of the boundaries 1 and 3 are zero. (b) Even symmetry in which voltages of all ${ }_{55}$ points of the grid of the structure are equal because of the oc even symmetry effect.

$45 \mathrm{c}$

16) As mentioned, by calculating the divergence of the sc obtained potentials or electrical fields, the capacitance of soc the structure can be easily obtained by summing the value ${ }^{5 c}$ of electrical fields across the boundaries. In that case,

${ }^{20}$ knowing the value of the permittivity of the liquid used for

${ }^{\text {sc }}$ our experimental Laplace equation solution is important.

17) If the capacitance of the structure is measured with another method, the value of the permittivity of liquid can be calculated.

18) From calculation of electrical fields which is equal to the derivative of the potentials obtained from the Laplace equation, it can be understood that the resulted voltages and electric field are independent from the liquid permittivity.

19) The electrical field lines and equipotential lines can be obtained by analyzing the potentials in MATLAB software. But the equipotential lines can be obtained directly from experimental solution by following the lines on which the value of the voltage is constant along them. Also the electrical field lines are traced by following the lines on which the values of the voltages have the maximum variations.

20) After all, this fact should be mentioned that all of the experiments have been done here, can be extended to a different 2D or a 3D Laplace problem such as a cubic or 
Vol. 5, Issue 9, September 2016

pyramidal 3D Laplace problem. Fig. 10 shows an example of 3D Laplace problem that can be solved with experimental, numerical or analytical methods.

\section{Conclusion}

Here, a very simple experimental setup was introduced to measure the electric potential of a 2D structure. Based on this setup, several classical electromagnetics theorems have been practically verified. These include, influence of boundaries values, mean value theorem, capacitance measurement, It is believed that the results of this research can be used as a tutorial tool for undergraduate students in Physics, Electromagnetics, and even in engineering mathematics course.

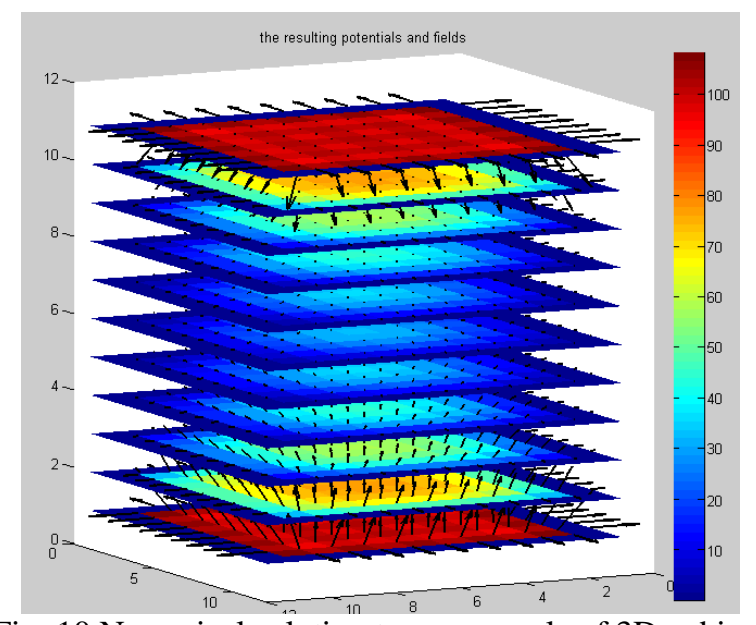

Fig. 10.Numerical solution to an example of 3D cubic Laplace problem

\section{ACKNOWLEDGMENT}

The authors would like to thank Amirkabir University of Technology for supporting this project. They specially thank Mr. Ahmad Naseri-Ali-Abadi for performing some of tests.

\section{REFERENCES}

[1] G. E. Urroz, "Introduction to Partial Differential Equations", September 2004.

[2] A. I. Prilenko, and E. D. Solomentsev, "Potential theory", Encyclopedia of Mathematics, 2001.

[3] M. N. O. Sadiku, "Numerical Technics in Electromagnetics", CRC Press, 2000.

[4] J. Cranka1 and P. Nicolson, "A practical method for numerical evaluation of solutions of partial differential equations of the heatconduction type," Mathematical Proceedings of the Cambridge Philosophical Society, vol. 43, Issue 1, pp. 50-67, 1947

[5] C Pozrikidis, "A practical guide to boundary element methods with the software library BEMLIB", Chapman \& Hall, 2002.

[6] H Ghassemi, S Panahi, AR Kohansal, Solving the Laplace's Equation by the FDM and BEM Using Mixed Boundary Conditions, American Journal of Applied Mathematics and Statistics vol. 4 (2016), 37-42

[7] P Li, F. Stagnitti and NF Uren, Analytical solution for finite domain Laplacian equation with coexistent boundary conditions, Mathematical and Computer Modelling, vol. 25, No. 10, (1997), $29-45$

[8] SB Gueye, K Talla, C Mbow, Solution of 1D Poisson Equation with Neumann-Dirichlet and Dirichlet-Neumann Boundary
Conditions Using the Finite Difference Method, Journal of Electromagnetic Analysis and Applications, 2014,vol. 6, 309-318

[9] Greenberg MD (2011) Advanced Engineering Mathematics. Prentice-Hall

[10] D Zill, WS Wright, MR Cullen (2011) Advanced Engineering Mathematics. Jones and Bartlett Learning, Burlington.

\section{BIOGRAPHY}

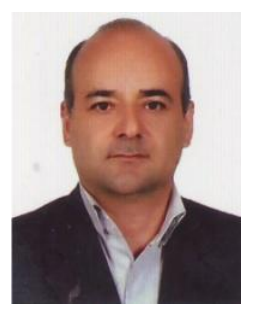

Gholamreza Moradi was born in Shahriar, Iran in 1966. He received his B.Sc. in Electrical Communication Engineering from Tehran University, Tehran, Iran in 1989, and the M.Sc. in the same field from Iran University of Science and Technology in 1993. Then he received his $\mathrm{PhD}$ degree in Electrical Engineering from Tehran Polytechnic University, Tehran, Iran in 2002. His research areas include applied and numerical Electromagnetics, Microwave measurement and antenna. He has published more than one hundred journal or conference papers and has authored/coauthored five books in his major. He is currently an associate professor with the electrical engineering department, Amirkabir University of Technology (Tehran Polytechnic), Tehran, Iran.

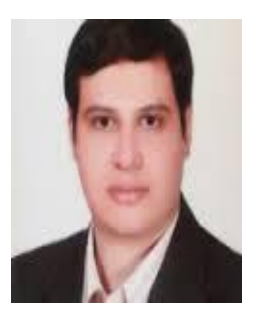

Mohammad Fater Marvasti received B.Sc. in Amirkabir University of Technology (Tehran Polytechnic), Tehran, Iran, both in electrical engineering, in 2016. He is currently working toward M.Sc. degree of Electrical Engineering in Sharif University of Technology. His current research interests include analysis and modeling of high frequency circuits. 